\title{
Neuromuscular Jumping Performance and Upper- Body Horizontal Power of Volleyball Players
}

\author{
Carlota A. Gonçalves, ${ }^{1}$ Tiago J.D. Lopes, ${ }^{1,2}$ Célia Nunes, ${ }^{3,4}$ Daniel A. Marinho, ${ }^{1,2}$ and Henrique P. Neiva ${ }^{1,2}$ \\ ${ }^{1}$ Department of Sport Sciences, University of Beira Interior, Covilhã, Portugal; ${ }^{2}$ Research Center in Sport Sciences, Health Sciences \\ and Human Development, CIDESD, Covilhã, Portugal; ${ }^{3}$ Department of Mathematics, University of Beira Interior, Covilhã, Portugal; and \\ ${ }^{4}$ Center of Mathematics and Applications, CMA-UBI, Covilhã, Portugal
}

\begin{abstract}
Gonçalves, CA, Lopes, TJD, Nunes, C, Marinho, DA, and Neiva, HP. Neuromuscular jumping performance and upper-body horizontal power of volleyball players. J Strength Cond Res XX(X): 000-000, 2019-The aim of the current study was to characterize the neuromuscular jumping performance and upper-body horizontal power of elite and subelite volleyball players. In addition, those neuromuscular performances were compared between field positions. One hundred twenty-two male volleyball players participated in the study: 83 elite players (mean $\pm S D: 24.11 \pm 5.57$ years) and 39 subelite players ( $25.38 \pm 6.19$ years). They were divided according to their playing position: setters $(n=22)$, opposite hitters $(n=16)$, middle hitters $(n=30)$, right-side hitters $(n=38)$, and liberos $(n=16)$. Each participant randomly performed 3 repetitions of medicine ball throwing (MBT), countermovement jump (CMJ), CMJ with free arms (CMJFA), and spike jump (SPJ). The results showed no significant differences between positions in the analyzed variables. However, there were differences between elite and subelite in the CMJ $\left(p=0.000, \eta_{p}^{2}=0.12\right)$, the CMJFA $\left(p=0.000, \eta_{p}^{2}\right.$ $=0.15)$, the SPJ $\left(p=0.000, \eta_{p}^{2}=0.21\right)$, and MBT $\left(p=0.001, \eta_{p}^{2}=0.09\right)$, showing a tendency for increased jumping performance and upper-body horizontal power for elite players. The elite opposite hitters and right-side hitters recorded greater CMJ performances ( $d=1.20$ and $d=1.62$, respectively). The right-side hitters were the only group that showed greater horizontal upper-body muscular power $(p=0.000, d=1.50)$. It is suggested that jumping performance is a determining factor for higher-level players, which was more relevant for the opposite hitters and right-side hitters. Nevertheless, the movement pattern of MBT seems to be relevant for the right-side hitters. Coaches should seek to develop jumping ability for the improvement of volleyball performances, without disregarding upper-body performances, depending on the position-specific demands.
\end{abstract}

Key Words: strength, countermovement jump, medicine ball

\section{Introduction}

Volleyball is a complex sport that depends on technical, tactical, and physical abilities (6). In the literature, several assumptions of sports performance in volleyball are well described, such as the anthropometric characteristics. An anthropometric profile of volleyball players has already been established, considering the specific anthropometric characteristics (height, arm length, palm length, as well as circumference of the ankle, calf, forearm, and arm). This is an important indicator of the potential of a player and a determining characteristic for the individual and collective success (10). Nevertheless, we should acknowledge that volleyball is characterized by several ballistic efforts such as vertical jumps, shots, and rapid changes of direction. Thus, upper-body horizontal power and neuromuscular jumping performance might play an important role in team success and should be relevant for the physical preparation of the player (14).

The relevance of muscular power to the volleyball player is supported by several studies, which focused on the impact of strength training in performance. Different resistance training programs, conducted for 5-8 weeks, found positive changes in the muscular power of the lower limbs of volleyball players $(14,20)$. Jumping ability seems to be a critical aspect for the player's performance (17). Recently, there have been more studies trying to

Address correspondence to Dr. Henrique P. Neiva, henriquepn@gmail.com. Journal of Strength and Conditioning Research 00(00)/1-6

(c) 2019 National Strength and Conditioning Association understand the effects of high-velocity strength training, such as plyometric training, on jumping performance in volleyball players, which have reported positive effects $(13,15,16,19,26,35,37)$. These studies showed the importance of muscular power of the lower limbs. However, only few studies focused on analyzing typical characteristics of the volleyball player and specifically regarding each player's function in the game.

A good volleyball player must be able to express the strength needed to block, jump, quickly react to the game context, adequately attack the ball, and properly answer to the variety of constraints in prolonged matches $(5,7)$. In this context, vertical jumping plays a major role. This physical characteristic supports other technical abilities and specific motor actions, as the block, the pass in suspension and the service in suspension $(12,28)$. However, we must acknowledge the importance of the upper-body horizontal power for the volleyball player (18). The muscular strength of the upper limbs seems to be crucial for the technical skills, such as the pass, the shot, and the service (22). In addition, there could exist a positive influence of the upper limbs' swing on the effect of the take off in jumping during the game (12). Although the analysis of the muscular power of the lower limbs has been widely investigated, few studies have so far investigated the upper-body power performance $(9,13,18)$.

It becomes evident that upper and lower neuromuscular power performances are essential in volleyball, but there is limited information available on the neuromuscular performance characteristics in male volleyball players according to field position and levels, mostly based on the analysis of samples with few subjects $(18,19,24,32)$. 
Research should focus on the requirements of higher performance for each position and level, supported on large number of subjects that provide clear evidences and conclusions. Therefore, the primary aim of the current study was to characterize the upper-body horizontal power and neuromuscular jumping performance in volleyball players, comparing the different levels (elite vs. subelite) and different players' position. The primary outcomes of our study are countermovement jump (CMJ), CMJ with free arms (CMJFA) and spike jump (SPJ) heights, and medicine ball throwing (MBT) horizontal distances. It was hypothesized that elite players and attackers would achieve higher values of upper-body horizontal power and neuromuscular jumping performance comparing with subelite and other players' positions.

\section{Methods}

\section{Experimental Approach to the Problem}

This study evaluated the upper-body and lower muscular power performances of volleyball players, characterizing the demands of each position, specifically the setters, middle hitters, opposite hitters, right-side hitters, and liberos. For this, a CMJ, a CMJFA, a SPJ, and MBT were evaluated. These tests are valid and reliable measurements of neuromuscular performance for both lower and upper limbs $(1,18,23,33)$. Each player performed 3 repetitions of each test, after a traditional warm-up (30), in a randomized order. Players were also divided by the elite and subelite level and according to usual position in the field to understand the different requirements of the player level and the position during the game. The procedures took place at one single session, but rest was guaranteed to all participants between tests so that performance was not compromised.

\section{Subjects}

One hundred twenty-two male national and international volleyball players (age: $24.52 \pm 5.78$ years, body mass: $80.07 \pm 9.64 \mathrm{~kg}$, height: $1.86 \pm 0.08 \mathrm{~m}$, and experience: $12.00 \pm 6.44$ years of practice) volunteered to participate in the current study. These players were part of 14 teams participating in the Portuguese national championships. The players were divided into elite and subelite levels according to their participation in the main national championship league (first Portuguese league) or in a lower-level league (second Portuguese league). Hence, 83 players were classified as the elite group (age: $24.11 \pm 5.57$ years, body mass: $81.23 \pm 9.09 \mathrm{~kg}$, and height: $1.88 \pm 0.08 \mathrm{~m}$ ). From this group, it is relevant to report that more than 50 subjects have already played in international competitions. The subelite level comprised 39 players (age: $25.38 \pm 6.19$ years, body mass: $77.61 \pm 10.42$ $\mathrm{kg}$, and height: $1.82 \pm 0.08 \mathrm{~m})$. All athletes were between 18 and 35 years old, and all subject characteristics were measured mean $\pm S D$. Athletes were familiarized with the protocol and periodically performed the evaluated exercises in their training sessions. In the 24-hour period before performing the tests, the subjects did not engage in any activity that was considered fatiguing. All the participants provided written informed consent to the experimental procedures after the possible benefits and risks of participation were explained to them. The investigation was conducted in accordance with the Declaration of Helsinki and was approved by the University of Beira Interior Research Ethics Committee (Table 1).

\section{Procedures}

The subjects were evaluated at the same time of the day for each participant under the same environmental conditions $\left(\sim 20^{\circ} \mathrm{C}\right.$ and $\sim 60 \%$ humidity). As the participants were competitive athletes, typically training more than 20 hours per week, the tests were accomplished on the day following a complete rest day, for 5 weeks (maximum of 12 subjects per day).

After arriving at the evaluation site, the participants were asked to sit comfortably, and after 5 minutes of rest, each player answered a small questionnaire to characterize their level and experience. Then, anthropometric measurements were performed. Body mass and height (Seca Instruments, Ltd., Hamburg, Germany) were measured before the warm-up protocol in the first testing session. Then, after 5 minutes, neuromuscular performance was assessed. The head coach and the assistant coach helped the researchers during the evaluation to guarantee the correct accomplishment of the tests.

Neuromuscular Performance:Vertical Jump Tests. For the evaluation of neuromuscular performance of the lower limbs, jump height was measured during a CMJ, a CMJFA, and a SPJ. After the usual warm-up, subjects performed 3 jumps separated by 2 minutes of rest, using an optical measurement system consisting of 2 transmitting and receiving cells (Optojump Next; microgate, Bolzano, Italy). Each transmission bar contained 96 LED lights (resolution of $1.0416 \mathrm{~cm}$ ) making continuous communication with the receiving cell and measuring contact times during jumps with an accuracy of 1/1,000th of a second.

Each CMJ evaluation trial started with the subjects standing with their knees fully extended and the hands on the hips to eliminate the influence of arm swing. They were then instructed to descend to a self-selected countermovement depth and to jump as high and quickly as possible. During the CMJFA, the arms were moved backward and forward energetically synchronized with leg flexion-extension movement (1). Also, in the CMJ, no horizontal approach was allowed, whereas in the SPJ, an approach of 3 or more steps was used in addition to arm movement simulating a volleyball spike (1). The mean value and the best value were considered for further analysis. The reliability of vertical performance was determined by the intraclass correlation coefficient (ICC), with mean values of 0.91 (IC 95\%: 0.88-0.94) for the CMJ, 0.93 (IC 95\%: 0.90-0.95) for the CMJFA, and 0.92 (IC 95\%: 0.86-0.95) for the SPJ, respectively. The coefficients of variation $(\mathrm{CVs})$ were $3.32 \%$ for the CMJ, $3.25 \%$ for the CMJFA, and $3.48 \%$ for the SPJ, respectively.

The combination of the vertical jumps described allowed for calculating some useful indexes of physical performance. The arm contribution index (AI) describes the percentage difference between CMJFA and CMJ heights (1):

$$
\mathrm{Al}(\%)=\frac{\mathrm{CMJFA}-\mathrm{CMJ}}{\mathrm{CMJ}} \times 100 .
$$

The approach index (API) is assessed based on the difference between the CMJFA and the SPJ (1):

$$
\text { API }(\%)=\frac{\text { SPJ }- \text { CMJFA }}{\text { CMJFA }} \times 100 \text {. }
$$

The AI allows the researchers to verify the contribution of the arms in the vertical jump, and the API would reveal the additional contribution of the horizontal approach on vertical jumping abilities.

Medicine Ball Throw. After assessing the lower limbs, each participant performed the evaluation of the upper-body horizontal power. The ball throwing performance (MBT) was measured by the horizontal distance reached after throwing a 3-kg ball 
Table 1

Characterization of the participants by player positions.*

\begin{tabular}{lcccc}
\hline Player position & No & Age $(\mathbf{M} \pm \mathbf{S D})$ & Stature $(\mathbf{m}) \mathbf{( M} \pm \mathbf{S D})$ & Body mass $(\mathbf{k g}) \mathbf{( M} \pm \mathbf{S D})$ \\
\hline 1 (setter) & 22 & $23.36 \pm 6.54$ & $1.85 \pm 0.06$ & $78.09 \pm 10.03$ \\
2 (opposite hitter) & 16 & $23.63 \pm 5.35$ & $1.87 \pm 0.05$ & $84.73 \pm 9.41$ \\
3 (middle hitter) & 30 & $25.07 \pm 5.13$ & $1.92 \pm 0.08$ & $83.44 \pm 8.18$ \\
4 (right-side hitter) & 38 & $24.89 \pm 6.66$ & $1.86 \pm 0.07$ & $77.73 \pm 8.71$ \\
Libero & 16 & $25.06 \pm 4.07$ & $1.79 \pm 0.07$ & $77.42 \pm 11.44$ \\
\hline
\end{tabular}

${ }^{\star} \mathrm{M}=$ means.

(circumference of $67 \mathrm{~cm}$ ). For the evaluation, each subject sat on the floor with his back against a rectilinear structure (wall). Each participant held the ball in front of him with both hands (close to the chest) to achieve the greatest breadth, speed, and distance as possible. All participants were instructed to prohibit rotation of the torso and the hip during the execution of the movement. Three attempts were counted with the $3-\mathrm{kg}$ medical ball, with a rest period of 1 minute between each throw. The distance in meters on each pitch and subject was counted. Overall, the MBT performance showed an average ICC of 0.958 (IC 95\%: 0.940-0.970), and the $\mathrm{CV}$ values were $2.98 \%$.

\section{Statistical Analyses}

Standard statistical procedures were selected to calculate means and SDs. The statistical procedures were performed using IBM SPSS Statistics for Windows, version 22.0 (IBM Corp., Armonk, NY, USA), and the level of statistical significance was set at $p \leq$ 0.05 . The normality and homogeneity of variance were confirmed by the Kolmogorov-Smirnov test $(n>30)$, and parametric tests were used to analyze the data. Reliability was measured by $\mathrm{CV}$ and ICC in the 3 trials conducted during the elite and subelite evaluation; this was calculated using the bidirectional mixed random effects model (absolute agreement type). A 2-way analysis of variance was conducted on the influence of 2 independent variables (player level and player position) on the dependent variables (CMJ, CMJFA, SPJ, AI, and API). Specific player positions included 5 levels (setters, middle hitter, opposite hitter, right-side hitter, and libero) and the player level consisted of 2 levels (elite and subelite). When a significant $F$ value was achieved, the Bonferroni post hoc procedure was performed to locate the pairwise differences. Effect size was calculated to estimate variance between players' positions and levels (partial eta squared: $\eta_{\mathrm{p}}^{2}$ ) and Cohen's $d(d)$ for comparisons between levels. $d$ values between 0.20 and 0.60 were considered small, between 0.60 and 1.20 were considered moderate, between 1.20 and 2.00 large, and $\geq 2.0$ were considered very large (11). For $\eta_{p}^{2}$, cutoff values were interpreted as 0.02 for small, 0.13 for moderate, and 0.26 for large (2).

\section{Results}

The results showed no interaction between specific playing position and level for all the variables (Table 2). Also, no significant interaction was found between the positions $(p>0.05)$. However, when comparing elite vs. subelite, significant differences were shown, revealing moderate effects for jumping performance $\left(0.11<\eta_{p}^{2}<0.22\right)$ and small effects for MBT $\left(\eta_{p}^{2}=0.09\right)$. In fact,

Table 2

Comparison of player positions in the variables of explosive strength of the upper and lower limbs. ${ }^{*} \dagger$

\begin{tabular}{|c|c|c|c|c|c|c|c|c|c|}
\hline \multirow[b]{2}{*}{ Variables } & \multirow[b]{2}{*}{ Groups } & \multirow[b]{2}{*}{ Setter } & \multirow[b]{2}{*}{ Opposite hitter } & \multirow[b]{2}{*}{ Middle hitter } & \multirow[b]{2}{*}{ Right-side hitter } & \multirow[b]{2}{*}{ Libero } & \multicolumn{3}{|c|}{ Two-way ANOVA $\left(\eta_{p}^{2}\right)$} \\
\hline & & & & & & & Position & Level & Positionł level \\
\hline \multirow[t]{3}{*}{$\overline{\mathrm{CMJ}(\mathrm{cm})}$} & Elite & $43.40 \pm 5.93$ & $46.51 \pm 6.56$ & $44.19 \pm 7.37$ & $47.93 \pm 5.67$ & $42.35 \pm 6.36$ & $p=0.45$ & $p=0.000 \S$ & $p=0.40$ \\
\hline & Subelite & $41.83 \pm 8.34$ & $39.59 \pm 5.21$ & $40.35 \pm 6.04$ & $39.73 \pm 4.07$ & $37.50 \pm 5.55$ & $(0.03)$ & $(0.12)$ & $(0.04)$ \\
\hline & $p$ value $[d]$ & $0.60[0.24]$ & $0.03[1.20] \ddagger$ & $0.11[0.54]$ & $0.000[1.62] \S$ & $0.18[0.86]$ & & & \\
\hline \multirow[t]{3}{*}{ CMJFA (cm) } & Elite & $50.95 \pm 8.12$ & $54.44 \pm 6.83$ & $52.75 \pm 8.78$ & $55.62 \pm 5.54$ & $49.16 \pm 6.51$ & $p=0.40$ & $p=0.000 \S$ & $p=0.32$ \\
\hline & Subelite & $49.50 \pm 10.98$ & $46.49 \pm 2.80$ & $46.58 \pm 7.64$ & $45.27 \pm 5.02$ & $42.68 \pm 5.47$ & $(0.04)$ & $(0.15)$ & $(0.04)$ \\
\hline & $p$ value $[d]$ & $0.67[0.17]$ & 0.03 [2.20]‡ & $0.03[0.76] \ddagger$ & $0.000[1.99] \S$ & $0.12[1.10]$ & & & \\
\hline \multirow[t]{3}{*}{ SPJ (cm) } & Elite & $62.57 \pm 9.52$ & $66.90 \pm 7.33$ & $63.66 \pm 9.02$ & $66.82 \pm 6.64$ & $60.16 \pm 7.74$ & $p=0.26$ & $p=0.000 \S$ & $p=0.79$ \\
\hline & Subelite & $57.15 \pm 11.20$ & $54.34 \pm 4.63$ & $55.03 \pm 9.70$ & $56.75 \pm 7.04$ & $50.93 \pm 9.45$ & $(0.05)$ & $(0.21)$ & $(0.02)$ \\
\hline & $p$ value $[d]$ & $0.17[0.57]$ & $0.003[2.04] \S$ & $0.01[0.97] \S$ & $0.001[1.54] \S$ & $0.05[1.21] \ddagger$ & & & \\
\hline \multirow[t]{3}{*}{ MBT (m) } & Elite & $6.35 \pm 0.38$ & $6.75 \pm 0.56$ & $6.15 \pm 0.88$ & $6.53 \pm 0.77$ & $6.09 \pm 0.57$ & $p=0.08$ & $p=0.001 \S$ & $p=0.12$ \\
\hline & Subelite & $6.22 \pm 0.69$ & $6.21 \pm 0.52$ & $5.92 \pm 0.57$ & $5.52 \pm 0.47$ & $5.67 \pm 0.69$ & $(0.07)$ & $(0.09)$ & $(0.06)$ \\
\hline & $p$ value $[d]$ & $0.65[0.39]$ & $0.10[1.06]$ & $0.37[0.27]$ & $0.000[1.50] \S$ & $0.27[0.76]$ & & & \\
\hline \multirow[t]{3}{*}{ Al (\%) } & Elite & $17.4 \pm 10.3$ & $17.5 \pm 7.3$ & $19.8 \pm 9.8$ & $16.4 \pm 5.2$ & $16.6 \pm 7.8$ & $p=0.39$ & $p=0.69$ & $p=0.82$ \\
\hline & Subelite & $17.7 \pm 7.8$ & $18.8 \pm 13.3$ & $15.4 \pm 7.6$ & $14.1 \pm 7.1$ & $14.2 \pm 7.6$ & $(0.01)$ & $(0.02)$ & $(0.01)$ \\
\hline & $p$ value $[d]$ & $0.96[0.03]$ & $0.80[0.14]$ & $0.23[0.50]$ & $0.27[0.40]$ & 0.60 [0.33] & & & \\
\hline \multirow[t]{3}{*}{ API (\%) } & Elite & $23.8 \pm 13.9$ & $23.3 \pm 7.4$ & $21.7 \pm 12.2$ & $20.4 \pm 8.0$ & $10.3 \pm 3.0$ & $p=0.16$ & $p=0.77$ & $p=0.21$ \\
\hline & Subelite & $16.5 \pm 8.4$ & $16.9 \pm 7.0$ & $18.1 \pm 8.5$ & $25.8 \pm 14.3$ & $9.3 \pm 4.6$ & $(0.18)$ & $(0.02)$ & $(0.05)$ \\
\hline & $p$ value $[d]$ & $0.16[0.60]$ & $0.10[0.95]$ & $0.41[0.34]$ & $0.14[0.54]$ & $0.51[0.41]$ & & & \\
\hline
\end{tabular}

*ANOVA = analysis of variance; $\mathrm{CMJ}=$ countermovement jump; CMJFA = countermovement jump free arms; SPJ = spike jump; MBT = medicine ball throw; $\mathrm{Al}=$ arm contribution index; API = approach index.

†Eta square $\left(\eta^{2}\right)$ was used to identify the effect size of the interaction; $p$ value and Cohen's $d[d]$ between levels are presented.

$\ddagger p \leq 0.05$.

$\S p \leq 0.01$. 
the results showed a tendency toward higher neuromuscular performance for the elite players in the different field positions. The only position that revealed no significant differences between players' level was the setter.

The greater magnitude of differences between elite and subelite players was found in the opposite and right-side hitters, with large and very large effects in the CMJ, CMJFA, and SPJ. Interestingly, MBT only showed to be different between player levels in the right-side hitter. In addition, the libero position only showed to be different between player levels regarding the SPJ, presenting large effect sizes. Moreover, despite the fact that the AI and API showed no interaction effects between players' positions and levels, the elite players tended to present higher values for the API with moderate effects for the setters and for the opposite hitters.

Figure 1 depicts the maximal values presented by the elite and subelite players in each position, for the neuromuscular variables assessed. One can clearly verify that elite-level volleyball players had a greater jump ability and upper-body power than subelite when collapsed across player positions. Interactions between the player level and position were found with the hitting positions.

\section{Discussion}

This study aimed to verify and to compare the upper-body horizontal power and neuromuscular jumping performance of elite and subelite volleyball players according to their specific positions during the game, thus seeking to characterize the different competitive levels and specificity of the volleyball player. It was verified that elite volleyball players performed better than the subelite level on all the muscular power variables for upper and lower limbs, with some particularities for each field player position. The right-side hitters showed the most relevant differences between players' levels. Several jumping performance variables were higher for the opposite (CMJ, CMJFA, and SPJ) and middle hitters (CMJFA and SPJ), whereas the elite-level libero was only clearly better in the SPJ. These results showed a tendency toward higher neuromuscular jumping performances by the higher-level volleyball players, with greater differences according to specific demands of the player position. Moreover, it seems that upperbody horizontal power is particularly relevant for better performances of right-side hitters.

The volleyball game is characterized by the high height of the game near the net, the velocity of players' responses to the game, the velocity of applied force on the ball, and the jumping ability required to efficiently perform these motor tasks for an improved performance (3). Some previous studies found that muscular power was a determining variable both for male and female volleyball players, suggesting the better players were those who could jump higher $(3,9)$. However, this neuromuscular performance seems to depend on the position during the game and on the specificity of each task demands (18). Yet, to the best of our knowledge, the current study was the first to provide a deeper study of neuromuscular power performance of the volleyball player, by extensively analyzing several jump-related variables and ballistic upper limb performances, comparing the players' levels and positions, using high-level players and a large number of participants. The values recorded from the upper and lower neuromuscular assessment in each position from nonelite players were quite similar to previous studies $(4,21)$. However, our elite players presented considerably higher performances than the previously reported values from other studies with elite players $(4,21,25)$.

As presented in Table 2, all the neuromuscular jumping performances were significantly higher in the elite players in all the field positions (except for the setters) with a moderate and large magnitude of effects compared with subelite players. These results are in accordance with previous findings $(18,31)$ and revealed the importance of developing vertical jumping skills in volleyball players for the CMJ, CMJFA, or SPJ. Previous research verified the
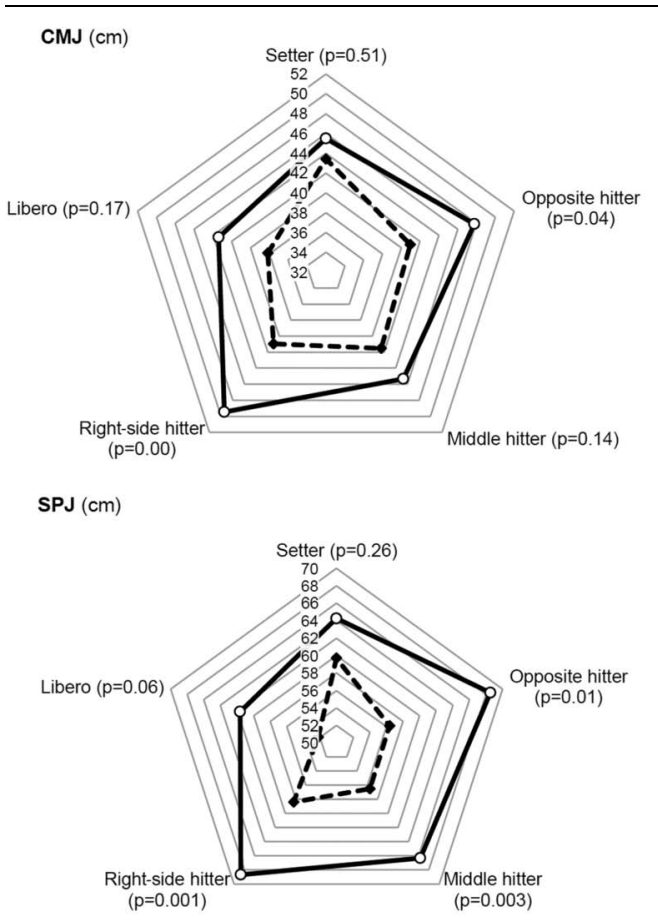
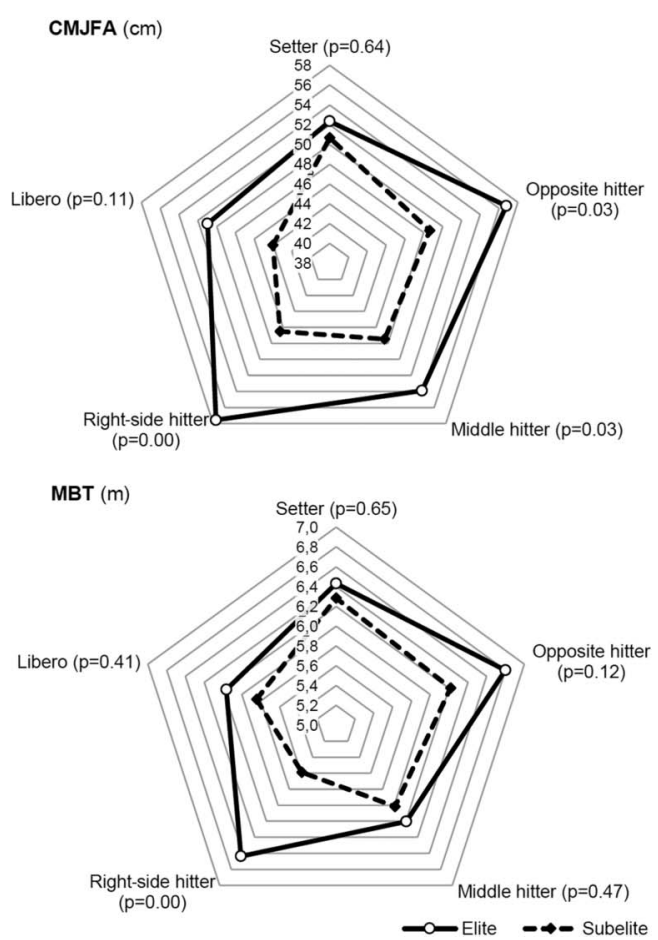

Figure 1. Maximal values of countermovement jump (CMJ), CMJ with free arms (CMJFA), spike jump (SPJ), and medicine ball throwing (MBT). Solid lines represent the elite level, and nonsolid lines represent the subelite level. 
importance of jumping ability to distinguish elite and subelite team players $(8,29,31)$, but only in college-level players. Indeed, it is evident that those differences exist for more experienced players. The current study clearly demonstrates the importance of the jumping ability in elite players, reinforcing the role of the jumping ability for a given movement required $(26,27)$. Nevertheless, it was interesting to notice that no differences between elite and nonelite players were found in the setters, thus suggesting neuromuscular performances are not a clear determining factor for the success on this position. The setters are usually skilled players used to set-up attacks that are completed by other players, determining they are involved in several game tasks mostly related with technical skills and not necessarily related with maximal neuromuscular performances $(8,18,34,36)$. Hence, probably the players' level would be determined by their technical ability, positioning, game perception, and set-up ability, rather than by their muscular performances (34).

As described in Table 2, and highlighted in Figure 1, the ballistic performance of the upper limbs in the elite group was higher than in the subelite group. Medicine ball throwing seems to be one of the variables overlooked in the volleyball-related research literature, with few studies on the evaluation of upper-body horizontal power $(18,19,32)$. Many studies reported the importance of muscular power of the lower limbs as described earlier, although they did not focus on the importance of the upper-body horizontal power in volleyball $(9,19,28)$. Our results are in accordance with previous data, showing the players from the first division differed from the second division by the higher MBT performance, as well as the better jumping performance (6).

Regarding the positions of the players during the game, no significant differences were found between them in each performance level, as showed in previous reports $(5,18)$. Figure 1 allows one to better understand the players' profiles and clarifies the trends for differences between players' levels and positions regarding the neuromuscular jumping performance and upper-body horizontal power. Although there were no significant differences between positions, it should be noted that right-side hitters showed the best jumping performance, specifically in the CMJ, CMJFA, and SPJ, whereas opposite hitters obtained the best results in MBT. At the highest competition level, the opposite hitters and right-side hitters are those with greater activity demands in a volleyball team. Moreover, the elite right-side hitters were the only position that revealed higher performances in all assessed variables, when compared with subelite levels. These players, owing to their technical and tactical roles, are focused in attacking tasks, thus it would be expected to find some performance differences (21). The rightside hitters are required to have better jumping ability because of their great jumping and attacking actions during the game $(6,21)$.

It should be noted that our study showed that setters and liberos were those with weaker performances. These results are in accordance with previous findings, reporting the MBT lowest performances for the libero position (18). This could be caused by the lack of upper-body-specific activities during competition. Along with the setters, these players revealed the weakest results, perhaps due to the limited involvement during competition. For instance, the libero players are required to be faster to move from side to side, but they are not great jumpers and the setters are not players who jump and use the ball shots so often during a game (25). Moreover, the setters are usually characterized by lower anthropometric characteristics (height and body mass), and these characteristics are related with lower CMJ and MBT performances (18). The libero and the setters need to be better at passing and throw the ball in a quick and objective manner, required to be mostly technical-tactical players.
No differences were found in both jumping indexes (AI and API), but moderate effects were found in the API for the right-side hitters and for the AI for the middle hitters, between elite and subelite levels. As expected, this result shows a tendency for the arm contribution to a higher vertical jump for the middle hitters, due to the constant demands of jumping from behind during the game in these players. So, we can suggest that the middle hitters are the most efficient players using the auxiliary movement of the arms for balance and improve jumping performance. In addition, the right-side hitters were showed to be more efficient in using the horizontal approach for higher jumps. These players usually perform spike jumping during matches and sometimes from behind, so these results were also expected.

We should acknowledge some limitations that should be considered when analyzing the current study results. A larger sample for the lower-level players would provide evidences with higher statistical power and lower margin of error. Also, more upperbody performance evaluations should be performed to better understand upper-body neuromuscular maximal performance characteristics of volleyball players. Moreover, the evaluations were performed in an intermediate stage of the season and with a competition schedule that could possibly influence some of the results. Hence, one could suggest that future studies should collect data in different times of the season.

In summary, our results suggested that neuromuscular jumping performance presents an essential role in volleyball players, especially for opposite hitters and right-side hitters. The upper-body horizontal power performance is suggested to determine the volleyball player level regarding right-side hitters. Nevertheless, it seems upper-body horizontal power could not be disregarded in other positions, such as the opposite hitters. Thus, this neuromuscular capability should also be improved for greater performances during the match.

\section{Practical Applications}

The assessment of the ability to jump and ball throwing in different positions revealed the specific requirements of volleyball players' performance. It was suggested that the right-side hitters and opposite hitters have higher neuromuscular jumping performance requirements. Moreover, upper-body horizontal power is needed for a higher performance, mainly for the rightside hitters. Considering those results, coaches should seek to develop jumping ability, as one of the primary physical components in volleyball players, and also upper-body horizontal power for some specific positions, as the right-side hitters.

It is also expected that the variables analyzed, and the results obtained could be useful for providing information on identification of elite-level players, comparing and establishing goals for improving performance in each specific individual playing position. Moreover, sport scientists and conditioning professionals should consider the analyzed characteristics of volleyball players when designing individualized position-specific training programs.

\section{Acknowledgments}

Supported by national funds granted by FCT-Portuguese Foundation for Science and Technology, I.P. (UID/DTP/04045/2019)—and the European Fund for Regional Development (FEDER) allocated by European Union through the COMPETE 2020 Program (POCI-010145-FEDER-006969)—competitiveness and internationalization (POCI). 


\section{References}

1. Borràs X, Balius X, Drobnic F, and Galilea P. Vertical jump assessment on volleyball: A follow-up of three seasons of a high-level volleyball team. J Strength Cond Res 25: 1686-1694, 2011.

2. Cohen J. The T-Test for Means. In: 2nd, ed. Statistical Power Analysis for the Behavioral Sciences. Hillsdale, NJ: Lawrence Erlbaum, 1988. pp. 19-74.

3. Dopsaj M, Ćopić N, Nešić G, and Sikimić M. Jumping performance in elite female volleyball players relative to playing positions: A practical multidimensional assessment model. Serb J Sports Sci 6: 61-69, 2012.

4. Dyba W. Physiological and activity characteristics of volleyball. Volley Tech J 6: 33-51, 1982.

5. Fonseca CL, Roquetti PF, and Fernández JF. Analysis of the Anthropometric Profile of Players of the Brazilian Youth and Youth Volleyball Team. Int J Morphol 28: 1035-1041, 2010.

6. Forthomme B, Croisier JL, Ciccarone G, Crielaard JM, and Cloes M. Factors correlated with volleyball spike velocity. Am J Sports Med 33 1513-1519, 2005.

7. Gabbett T and Georgieff B. Physiological and anthropometric characteristics of Australian junior national, state, and novice volleyball players. J Strength Cond Res 21: 902-908, 2007.

8. Gabbett T, Georgieff B, and Domrow N. The use of physiological, anthropometric, and skill data to predict selection in a talent-identified junior volleyball squad. J Sport Sci 25: 1337-1344, 2007.

9. González Y, Sedano S, Fernández J, and Díaz H. Comparative study on Antropométric factors and physical condition in young Colombian Volleyball players. Revista UDCA Actualidad Divulgación Científica 17: 53-63, 2014.

10. Häkkinen K. Changes in physical fitness profile in female volleyball players during the competitive season. J Sport Med Phys Fitness 33: 223-232, 1993.

11. Hopkins WG, Marshall SW, Batterham AM, and Hanin J. Progressive statistics for studies in sports medicine and exercise science. Med Sci Sports Exerc 41: 3-13, 2009.

12. Kitamura K, Pereira LA, Kobal R, Cal Abad CC, Finotti R, Nakamura FY, et al. Loaded and unloaded jump performance of top-level volleyball players from different age categories. Biol Sport 34: 273-278, 2017.

13. Krističević T, Krakan I, and Baić M. Effects of short high impact plyometric training on jumping performance in female volleyball players. Acta Kinesiologica 10: 25-29, 2016.

14. Lehnert M, Sigmund M, Lipinska P, Vařeková R, Hroch M, Xaverova Z, et al. Training-induced changes in physical performance can be achieved without body mass reduction after eight week of strength and injury prevention oriented programme in volleyball female players. Biol Sport 34: 205-213, 2017.

15. Malatesta D, Cattaneo F, Dugnani S, and Maffiuletti NA. Effects of electromyostimulation training and volleyball practice on jumping ability. J Strength Cond Res 17: 573-579, 2003.

16. Markovic S, Mirkov DM, Knezevic OM, and Jaric S. Jump training with different loads: Effects on jumping performance and power output. Eur J Appl Physiol 113: 2511-2521, 2013.

17. Marques MC and Marinho DA. Physical parameters and performance values in starters and non-starters volleyball players: A brief research note. Motricidade 5: 7-11, 2009.

18. Marques MC, van den Tillaar R, Gabbett TJ, Reis VM, and GonzálezBadillo JJ. Physical fitness qualities of professional volleyball players: Determination of positional differences. J Strength Cond Res 23: 1106-1111, 2009.

19. Marques MC, Van Den Tillaar R, Vescovi JD, and González-Badillo JJ. Changes in strength and power performance in elite senior female professional volleyball players during the in-season: A case study. J Strength Cond Res 22: 1147-1155, 2008.

20. Pereira A, Costa AM, Santos P, Figueiredo T, and João PV. Training strategy of explosive strength in young female volleyball players. Medicina (Kaunas) 51: 126-131, 2015.

21. Polglaze T and Dawson B. The physiological requirements of the positions in state league volleyball. Sports Coach 15: 32, 1992.

22. Pu JZ, Gao CX, and Feng WQ. The Handbook of Function Evaluation for Elite Players. Beijing, China: Peoples' Sports Press, 1989. pp. 206-210.

23. Roe G, Shaw W, Darrall-Jones J, Phibbs PJ, Read D, Weakley JJ, et al. Reliability and validity of a medicine ball-contained accelerometer for measuring upper-body neuromuscular performance. J Strength Cond Res 32: 1915-1918, 2018.

24. Rousanoglou EN, Georgiadis GV, and Boudolos KD. Muscular strength and jumping performance relationships in young women athletes. J Strength Cond Res 22: 1375-1378, 2008.

25. Sheppard JM and Borgeaud R. Influence of stature on movement speed and repeated efforts in elite volleyball players. J Aust Strength Cond 16: 12-14, 2008.

26. Sheppard JM, Gabbett T, and Borgeaud R. Training repeated effort ability in national team male volleyball players. Int J Sport Physiol Perform 3: 397-400, 2008.

27. Sheppard JM, Gabbett T, Taylor KL, Dorman J, Lebedew AJ, and Borgeaud R. Development of a repeated-effort test for elite men's volleyball. Int J Sports Physiol Perform 2: 292-304, 2007.

28. Sheppard JM, Gabbett TJ, and Stanganelli LCR. An analysis of playing positions in elite men's volleyball: Considerations for competition demands and physiologic characteristics. J Strength Cond Res 23: 1858-1866, 2009.

29. Sheppard JM, Hobson S, Barker M, Taylor K, Chapman D, McGuigan M, et al. The effect of training with accentuated eccentric load counter-movement jumps on strength and power characteristics of high-performance volleyball players. Int J Sport Sci Coa 3: 355-363, 2008.

30. Silva LM, Neiva HP, Marques MC, Izquierdo M, and Marinho DA. Effects of warm-up, post-warm-up, and Re-Warm-Up strategies on explosive efforts in team sports: A systematic review. Sports Med 48: 2285-2299, 2018.

31. Smith D, Roberts D, and Watson B. Physical, physiological and performance differences between Canadian national team and universiade volleyball players. J Sport Sci 10: 131-138, 1992.

32. Stanganelli LCR, Dourado AC, Oncken P, Mançan S, and da Costa SC. Adaptations on jump capacity in Brazilian volleyball players prior to the under-19 World Championship. J Strength Cond Res 22: 741-749, 2008.

33. Tavares F, Simões M, Matos B, Smith TB, and Driller M. Wellness, muscle soreness and neuromuscular performance during a training week in volleyball athletes. J Sport Med Phys Fit 58: 1852-1858, 2018.

34. Thissen-Milder M and Mayhew J. Selection and classification of high school volleyball players from performance tests. J Sport Med Phys Fit 31: 380-384, 1991.

35. Trajković N, Krističević T, and Baić M. Effects of plyometric training on sport-specific tests in female volleyball players. Acta Kinesiologica 10: 20-24, 2016.

36. Viviani F and Casagrande G. Somatotype characteristics of Italian male basketball, soccer and volleyball players. J Sport Sci 8: 184, 1990.

37. Voelzke M, Stutzig N, Thorhauer HA, and Granacher U. Promoting lower extremity strength in elite volleyball players: Effects of two combined training methods. J Sci Med Sport 15: 457-462, 2012. 